\title{
OBSERVABLES IN GENERAL RELATIVITY
}

\author{
J.N. GoldberG* AND D.C. Robinson \\ Department of Mathematics, King's College, Strand, London WC2R 2LS, UK
}

\section{Dedicated to Prof. Iwo Bialynicki-Birula on the occasion of his 60th birthday}

We emphasize the importance of finding the "true" observables of the Einstein theory for canonical quantum gravity. The Hamilton-Jacobi theory for constrained systems is outlined and its application in terms of Ashtekar variables and Dirac variables is discussed. A specific example of a Bianchi II model is solved and the role of one variable as a "time" parameter is indicated. We end with a brief remark about an alternative approach.

PACS numbers: 04.60.+n, 04.20.Fy

\section{Introduction}

Iwo has always been interested in the fundamental problems of plysics. And so we have chosen to discuss one of the oldest and most perplexing problems in general relativity - the search for the observables. These observables are not necessarily measurable quantities. Measurable quantities, at least in a classical theory, are those quantities which can be evaluated in a coordinate and gauge invariant way relative to a measuring apparatus in a specified frame. The observables we are talking about, the so-called "true observables", are the independent degrees of freedom of the source free gravitational field [1-4]. The analogous quantities in electromagnetism are the transverse parts of the electromagnetic field which represent the data which uniquely describes the field independent of the gauge frame. For the classical theory, the search for these "true observables" is an interesting formal problem. For a quantum theory of gravity, it is a crucial problem. According to Dirac [5], only these observables or functions of them, should become quantum operators.

Recently there have been a number of proposals to deal with this problem by introducing auxiliary fields. Carlo Rovelli [6] introduces a congruence of observers - he calls them clocks - whose dynamics are introduced explicitly into the Lagrangian and Hamiltonian of the gravitational field. The clocks introduce intrinsic coordinates through their labels in a 3-dimensional label space and the proper time along their world lines. Karel Kuchar [7] accomplishes a similar result using a pressure free dust to establish an intrinsic frame. While both of these lead

*On leave from the Department of Physics, Syracuse University, Syracuse, NY 13244-1130. 
to interesting results, we want to stress the importance of finding the independent degrees of freedom for the empty space Einstein equations. In the following, we shall make some brief historical remarks relevant to our thinking. We shall then discuss some recent progress in the exact theory using the Hamilton-Jacobi formalism. But, we shall treat in detail only one of the Bianchi models in which the program can be performed completely. We will conclude with some remarks.

\section{Background}

The phase space of general relativity $\Gamma,\left(q_{a b}, p^{a b}\right)$ consists of positive definite 3-metrics on a space-like hypersurface $\Sigma$ and their canonical conjugates which are essentially the extrinsic curvatures of $\Sigma$ as embedded in 4-dimensional space-times

$$
\begin{gathered}
\Gamma: \quad\left(q_{a b}, p^{a b} ; \Sigma\right) \\
p^{a b}=-q^{\frac{1}{2}}\left(K^{a b}-q^{a b} K\right) .
\end{gathered}
$$

$K_{a b}$ is the extrinsic curvature and $K$ is its trace.

A point of the phase space represents possible initial data for a solution to the vacuum Einstein equations. However, because of the 4-dimensional diffeomorphisms which leave the Einstein equations invariant, suitable data satisfy four constraints per space point

$$
\begin{aligned}
& \mathcal{H}_{a}=-2 D_{b} p_{a}^{b}=0, \\
& \mathcal{H}_{\perp}=q^{-\frac{1}{2}}\left(p^{a b} p_{a b}-\frac{1}{2} p^{2}\right)-q^{\frac{1}{2} 3} R=0 .
\end{aligned}
$$

Up to a surface term, the Hamiltonian is a linear combination of the constraints,

$$
H=\int_{\Sigma}\left\{N H_{\perp}+N^{a} \mathcal{H}_{a}\right\} \mathrm{d}^{3} x,
$$

$N$ is the lapse function and $N^{a}-$ the shift vector.

Therefore, the physical phase space variables lie on a constraint surface $\Gamma_{\mathrm{C}}$ defined by $\mathcal{H}_{a}=\mathcal{H}_{\perp}=0$. The constraints

$$
C(N)=\int_{\Sigma} N^{a} \mathcal{H}_{a} \mathrm{~d}^{3} x,
$$

generate 3-dimensional mappings of $\Sigma \rightarrow \Sigma$ while

$$
C(N)=\int_{\Sigma} N H_{\perp} \mathrm{d}^{3} x
$$

generates mappings orthogonal to $\Sigma$. Therefore, at each point of the constraint surface, they generate a four parameter mapping of data for equivalent solutions. Thus $\Gamma_{\mathrm{C}}$ is divided into four parameter families of equivalence classes of initial data. The observables label equivalence classes and, therefore, are constant within each class. Thus, modulo the constraints themselves, the observables have vanishing Poisson brackets with the constraints. Starting with a phase space of 12 dimensions per space point, reducing to the constraint surface leaves 8 , and fully reducing to the phase space of equivalence classes leaves 4 dimensions per space point. Hence, a minimal set of observables will consist of 2 degrees of freedom per 
space point or four functions on the space-like surface $\Sigma$. According to Dirac, only the algebra of these four functions should become the operator algebra for the quantum theory.

One way to look for observables is to use the Hamilton-Jacobi formalism [8-11]. In an unconstrained system in classical mechanics, the Hamilton-Jacobi function, $S\left(q_{a}, \pi^{a} ; t\right), a=1 \ldots n$, is a solution of the equation

$$
H^{\prime}=H\left(q_{a}, \frac{\partial S}{\partial q_{a}}\right)+\frac{\partial S}{\partial t}=0 .
$$

$S\left(q_{a}, \pi^{a} ; t\right)$ is the generator of a canonical transformation $(q, p) \rightarrow(\gamma, \pi)$.

$$
p^{a}=\frac{\partial S}{\partial q_{a}}, \quad \gamma_{a}=\frac{\partial S}{\partial \pi^{a}}, \quad\left\{\gamma_{a}, \pi^{b}\right\}=\delta^{a}{ }_{b} .
$$

The new IIamiltonian is identically zero. Thus, the new coordinates and momenta $\left(\gamma_{a}, \pi^{a}\right)$ are constants of the motion. A solution of the dynamical problem is uniquely defined by these constants. Inversion of the transiomation gives the solution of the equations of motion in terms of the original variables

$$
q_{a}=q_{a}\left(\gamma_{b}, \pi^{b} ; t\right), \quad p^{a}=p^{a}\left(\gamma_{b}, \pi^{b} ; t\right) .
$$

Often the new variables are not measurable; for example, the action coordinate of the harmonic oscillator. Inversion of the transformation may then be necessary in order to construct measurable quantities.

When the system has first class constraints [9]:

$$
C_{i}\left(q_{a}, p^{a}\right)=0, \quad i=1 \ldots m, \quad\left\{C_{i}\left(q_{a}, p^{a}\right), H\right\}=\sum_{j=1}^{m} \Lambda_{i}^{j} C_{j}
$$

the Hamiltonian has the form

$$
H=H_{0}\left(q_{a}, p^{a}\right)+\sum_{i=1}^{m} \lambda^{i} C_{i} .
$$

Because the functions $\lambda^{i}$ are arbitrary, the propagation from the initial data is no longer unique. The Hamilton-Jacobi equation becomes, because the $\lambda^{i}$ are arbitrary, generalized to

$$
H_{0}\left(q_{a}, \frac{\partial S}{\partial q_{a}}\right)+\frac{\partial S}{\partial t}=0, \quad C_{i}\left(q_{a}, \frac{\partial S}{\partial q_{a}}\right)=0
$$

with $S=S\left(q_{a}, \pi^{A} ; t\right)$ and

$$
p^{a}=\frac{\partial S}{\partial q_{a}}, \quad \gamma_{A}=\frac{\partial S}{\partial \pi^{A}}, \quad A=1 \ldots n-m .
$$

As the constraints gencrate canonical transformations which leave the Hamiltonian invariant on the constraint surface, they generate the symmetry transformations of the dynamical system which divide the constraint surface into equivalence classes. In terms of the new variables, the constraints are identically satisfied. Therefore, the index $A=1 \ldots n-m$. The $\gamma_{A}$ and $\pi^{A}$ are constant within an equivalence class. These variables label a unique physical solution, but the inversion to the original variables is not unique. In fact, in order to perform the inversion, it 
is necessary to introduce $m$ parameters $f^{i}, i=1 \ldots m$, which may be arbitrary functions of the time

$$
q_{a}=q_{a}\left(\gamma_{A}, \pi^{A} ; f^{i}, t\right), \quad p^{a}=p^{a}\left(\gamma_{A}, \pi^{A} ; f^{i}, t\right) .
$$

The same is true if the Hamiltonian is just a linear combination of the constraints $-H_{0} \equiv 0$ :

$$
H=\sum_{i=1}^{m} \lambda^{i} C_{i}=0 .
$$

However, in this case it follows from (9) that there is no explicit time dependence. Therefore, the inversion also has no explicit time dependence. It may nonetheless be possible to define the evolution of the system in terms of one of the parameters introduced to effect the inversion.

\section{Exact theory}

Roughly two years ago, investigations using the Hamilton-Jacobi function was renewed in an interesting way, principally by Newman and Rovelli $[12,13]$. They make use of the Ashtekar formalism for general relativity so that the phase space is coordinated by the canonically conjugate variables $\left(A^{i}{ }_{a}, E_{i}{ }^{a}\right)$, an $\mathrm{SL}(2, \mathrm{C})$ connection and a densitized triad. In addition to the vector and scalar constraints there is now also an $\mathrm{SL}(2, \mathrm{C})$ gauge constraint, so the constraints are

$$
\begin{aligned}
& \mathcal{G}:=D_{a} E_{i}{ }^{a}=\partial_{a} E_{i}{ }^{a}+\eta_{i j k} A^{j}{ }_{a} E_{k}{ }^{a}=0, \\
& \mathcal{H}_{a}:=F^{i}{ }_{a b} E_{i}{ }^{b}=0, \\
& H_{\perp}:=F^{i}{ }_{a b} E_{j}{ }^{a} E_{k}{ }^{b} \eta_{i}{ }^{j k}=0, \\
& {F^{i}}^{i}{ }_{a b}=2 d A^{i}+\eta^{i}{ }_{j k} A^{j} \wedge A^{k} .
\end{aligned}
$$

The holonomy group of the connection is defined by the parallel transport around closed loops in $\Sigma$ :

$$
U[A, \alpha]=P \exp \oint_{\alpha} A^{i}{ }_{a} \tau_{i} \dot{\alpha} \dot{\alpha}_{1}^{a} \mathrm{~d} s
$$

where the $\tau_{i}$ are the Pauli matrices and $x^{a}=\alpha^{a}(s)$ is the closed curve. $U$ itself is clearly gauge covariant and its trace is gauge invariant. By making use of this property, Newman and Rovelli were able to find a Hamilton-Jacobi functional which satisfies the gauge constraint

$$
D_{a}\left(\frac{\delta S\left(A_{a}^{i}, u^{I}, v^{I}\right)}{\delta A^{i}{ }_{a}}\right)=0
$$

where $u^{I}$ and $v^{I}, I=1 \ldots 3$ are six scalar functions. Their canonical conjugates $q_{u I}, q_{v I}$ can be obtained explicitly from $S$. They then are able to satisfy the vector constraint by choosing $v^{I}$ to be intrinsic coordinates on $\Sigma$. In order to treat the scalar constraint one needs to express the connection and triad in terms of the new variables plus some arbitrary functions. That inversion has not been performed and it may not be possible to do so explicitly. 
We tried another approach. We work with the Dirac phase space $\left(q_{a b}, p^{a b}\right)$ and introduce the coefficients of the characteristic equation for the 3-dimensional Ricci tensor ${ }^{3} R_{a b}$,

$$
\operatorname{det}\left({ }^{3} R_{a}{ }^{b}-\lambda \delta_{a}{ }^{b}\right)=0
$$

as intrinsic coordinates on $\Sigma$. This yields the three scalars $R^{I}, I=1 \ldots 3$,

$$
R^{1}=R_{a}^{a}, \quad R^{2}=R_{b}^{a} R_{a}^{b}-\left(R_{a}^{a}\right)^{2}, \quad R^{3}=\operatorname{det}\left(R_{b}^{a}\right) .
$$

Three scalar densities are similarly formed from $p^{a b}, p^{I}$ :

$$
\begin{aligned}
& \operatorname{det}\left(p_{a}{ }^{b}-\omega \delta_{a}{ }^{b}\right)=0, \\
& p^{\underline{1}}=p_{a}^{a}, \quad p^{2}=p_{b}^{a} p_{a}^{b}-\left(p^{a}{ }_{a}\right)^{2}, \quad p^{3}=\operatorname{det}\left(p^{a}{ }_{b}\right) .
\end{aligned}
$$

The functions $P^{I}\left(R^{J}\right)$,

$$
\left(\operatorname{det}\left(R^{J}, a\right)\right)^{I} P^{I}\left(R^{J}\right)=p^{I}(x) \quad(\text { no sum on } I),
$$

are invariant under diffeomorphisms of $\Sigma$. Assuming that we can find a Hamilton -Jacobi functional $S\left(q_{a b}, P^{I}\right)$ which satisfies

$$
\begin{aligned}
& D_{b}\left(\frac{\partial S\left(q_{a b}, P^{I}\right)}{\partial q_{a b}}\right)=0, \\
& Q_{I}=\frac{\delta S}{\delta P^{I}}
\end{aligned}
$$

then we have found coordinates $\left(Q_{I}, P^{I}\right)$ for the partially reduced phase space. It then follows that the scalar constraint has a very simple algebraic form

$$
H_{\perp}=\mathrm{e}^{-\frac{3}{2} Q_{1}}\left(P^{\underline{2}}+\frac{1}{2}\left(P^{1}\right)^{2}\right)-\mathrm{e}^{\frac{3}{2} Q \underline{1}} R^{1}=0, \quad Q_{\underline{1}}=\operatorname{det}\left(q_{a b}\right) .
$$

The fully reduced phase space is easily constructed from this Hamiltonian. Rather than continue with this hypothetical solution, we shall present a finite dimensional model whose Hamiltonian has a similar algebraic structure.

\section{Bianchi II model}

We consider a homogeneous space-time, Bianchi type II, where only the time dependence of the dynamical variables remains. Therefore, the vector constraint is automatically satisfied and only the scalar constraint remains. In this case, the Hamiltonian has a similar algebraic form to that just mentioned and we can construct the solution to the Hamilton-Jacobi equation.

The metric is diagonal and following Misner one writes

$$
\begin{aligned}
& q_{a b}=(\exp 2 \beta)_{a b}, \\
& \left(\begin{array}{ccc}
\beta^{1} & & \\
& \beta^{2} & \\
& & \beta^{3}
\end{array}\right) .
\end{aligned}
$$

After the point transformation

$$
\beta^{1}=\sqrt{3}\left(\beta^{0}+\beta^{+}+\beta^{-}\right)
$$




$$
\begin{aligned}
& \beta^{2}=\sqrt{3}\left(\beta^{0}+\beta^{+}-\beta^{-}\right), \\
& \beta^{3}=-\sqrt{3} \beta^{+},
\end{aligned}
$$

the Hamiltonian becomes

$$
H=-\frac{1}{2}{p_{0}}^{2}+\frac{1}{2}{p_{+}}^{2}+\frac{1}{2}{p_{-}}^{2}+6 \mathrm{e}^{4 \sqrt{3} \beta^{+}}=0 .
$$

This system has a phase space of 6 dimensions with one constraint. Thus, there are four observables to be found. Three quantities with vanishing Poisson brackets with the Hamiltonian are immediately visible, $p_{0}, p_{-}$, and $p_{+}+12 \mathrm{e}^{4 \sqrt{3} \beta^{+}}$, but they are not independent. To find the Hamilton-Jacobi functional and the other two observables, introduce $P$ and $Q$ by

$$
\begin{aligned}
& P^{2}={p_{+}}^{2}+12 \mathrm{e}^{4 \sqrt{3} \beta^{+}} \\
& \Rightarrow \beta^{+}=\frac{1}{4 \sqrt{3}}\left[\ln \left(P^{2}-{p_{+}}^{2}\right)-\ln (12)\right], \\
& Q=\frac{\partial F}{\partial P}=\frac{\sqrt{3}}{12} \ln \frac{P-p_{+}}{P+p_{+}}, \\
& F=\frac{\sqrt{3}}{12}\left\{\left(P-p_{+}\right)\left[\ln \left(P-p_{+}\right)-1\right]\right. \\
& \left.\quad-\left(P+p_{+}\right)\left[\ln \left(P+p_{+}\right)-1\right]+p_{+} \ln (12)\right\} .
\end{aligned}
$$

where $F\left(p_{+}, P\right)$ is the generating function of the canonical transformation to $(Q, P)$. Now

$$
H=-\frac{1}{2}{p_{0}}^{2}+\frac{1}{2} P^{2}+\frac{1}{2} p_{-}^{2}=0 .
$$

We now consider a Hamilton-Jacobi function of the form $S=S\left(\beta^{0}, P, p_{-}\right)$ and write

$$
S=\beta^{0} \sqrt{P^{2}+p_{-}^{2}}-Q^{+} P-Q^{-} p_{-} .
$$

We find

$$
-\left(\frac{\partial S}{\partial \beta^{0}}\right)_{1}^{2}+P^{2}+p_{-}^{2} \equiv 0 .
$$

We see that the Hamiltonian is identically zero and the resulting canonical transformation yields

$$
\begin{aligned}
& p_{0}=\frac{\partial S}{\partial \beta^{0}}=\sqrt{P^{2}+p_{-}^{2}} \\
& Q=-\frac{\partial S}{\partial P}=Q^{+}-\frac{P}{\sqrt{P^{2}+p_{-}^{2}}} \beta^{0} \\
& \beta^{-}=-\frac{\partial S}{\partial p_{-}}=Q^{-}-\frac{p_{-}}{\sqrt{P^{2}+p_{-}^{2}}} \beta^{0}, \\
& P_{+}=-\frac{\partial S}{\partial Q^{+}}=P,
\end{aligned}
$$




$$
P_{-}=-\frac{\partial S}{\partial Q^{-}}=p_{-} .
$$

The observables on the reduced phase space are $\left(Q_{+}, Q_{-}, P^{+}, P^{-}\right)$. We note from the transformation that only $\beta^{0}$ is not determined. It is an arbitrary function of the time and can very well be interpreted to be the time itself.

The transition to quantum theory can now be made in the usual way by

$$
\left[Q^{A}, P_{B}\right]=\mathrm{i} \hbar \delta_{B}{ }^{A}, \quad A, B= \pm .
$$

All quantities are real so there are no reality conditions to apply and $Q_{ \pm}$and $P^{ \pm}$ are Hermitian. Note that since these operators are constants of the motion, they are Heisenberg operators. This point has been remarked on by Rovelli [14]. The physical state vectors are functions of a commuting set of the observables, $Q_{ \pm}$, for example, and the Hilbert space should be defined by square integrability with a measure defined by the requirement of Hermiticity. That discussion will take us beyond the scope of this talk.

Note, however, that the $\left(Q_{A}, P^{A}\right)$ are not obviously measurable quantities. To get back to such measurable quantities means inverting the transformations and expressing them in terms of these operators and $\beta^{0}$. In this way, an evolutionary parameter reenters the description. Thus we truly have a Heisenberg representation with constant state vectors and "time" dependent operators.

\section{Conclusions}

The point we wish to emphasize is that it is the geometry which tells us where we are and it is the geometry which gives a meaning to evolution or time propagation. In fact, the geometry may tell us that "where" and "when" break down and have no meaning. Wald [15], from a different point of view, has come to the same conclusion. He considers as an example the Bianchi IX model which has complicated phases of expansion and contraction. For his considerations, which are based on the Wheeler-DeWitt equation, time is meaningless near the transition points.

Recently, there have been some new developments along different lines. In particular, there is the work based on the new variables of Abhay Ashtekar [16] which makes use of the holonomy group. This appears to be moving in at least two directions. One is to generalize the algebra of the holonomy group and thereby obtain appropriate operators for the quantum theory $[17,18]$. The other is being developed principally by Rovelli and Smolin using loop variables to construct state vectors which satisfy the constraints [19]. We think that this work is very hopeful. These approaches in their own way are looking for the observables. Therefore, if one wants to consider the quantization of the Einstein theory, there is little choice but to try to find the observables.

Let us conclude by saying that this problem about observables was alive and kicking when Iwo began his career. It is still interesting and vital today. And so is he. 


\section{Acknowledgment}

The authors wish to thank SERC for support of J.N.G. by a SERC Visiting Fellowship Grant GR/H56472 during the summer and fall term of 1993. The work of J.N.G. was also supported in part by the National Science Foundation, Grant PHY 9005790. He is grateful for the hospitality of the Department of Mathematics at King's College.

\section{References}

[1] J.L. Anderson, P.G. Bergmann, Phys. Rev. 83, 1018 (1951).

[2] P.G. Bergmann, A. Komar, in: General Relativity and Gravitation, Vol. 1, Ed. A. Held, Plenum Press, New York 1980, p. 227.

[3] P.A.M. Dirac, Proc. R. Soc. Lond. A 246, 326 (1958).

[4] K. Kuchar, in: Quanlum Gravily 2, Eds. C.J. Isham, R. Penrose, D. Sciama, Clarendon Press, Oxford 1981, p. 329.

[5] P.A.M. Dirac, Lectures on Quantum Mechanics, Academic Press, New York 1967.

[6] C. Rovelli, Class. Quantum Grav. 8, 297 (1991).

[7] K. Kuchar, in: Brill-Misner Celebration, May 1993.

[8] P.G. Bergmann, Phys. Rev. 144, 1078 (1966).

[9] A. Komar, Phys. Rev. 170, 1175 (1968).

[10] A. Komar, Phys. Rev. D 1, 1521 (1970).

[11] C. Teitelboim, M. Henneaux, Quantization of Gauge Systems, Princeton University Press, Princeton 1992.

[12] J.N. Goldberg, E.T. Newman, C. Rovelli, J. Math. Phys. 32, 2739 (1991).

[13] E.T. Newman, C. Rovelli, Phys. Rev. Lett. 69, 1300 (1992).

[14] C. Rovelli, Phys. Rev. D 42, 2638 (1991).

[15] R. Wald, Phys. Rev. D 48, R2377 (1993).

[16] A. Ashtekar, Leclures on Non-perturbative Canonical Gravity, World Scientific, Singapore 1991.

[17] A. Ashtekar, C.J. Isham, Class. Quantum Grav. 9, 1433 (1992).

[18] A. Ashtekar, J. Lewandowski, in: Knots and Quantum Gravity, Ed. J. Baez, Oxford University Press, Oxford 1994, to appear. 